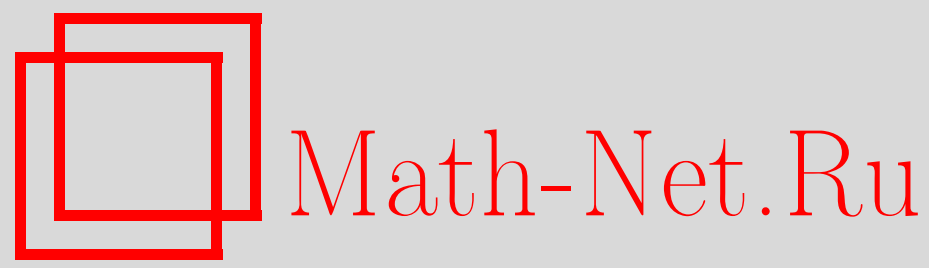

И. Р. Шафаревич, Семейства приводимых абелевых поверхностей, Матем. заметки, 1996, том 60, выпуск 6, 946-949

DOI: https://doi.org/10.4213/mzm1916

Использование Общероссийского математического портала MathNet.Ru подразумевает, что вы прочитали и согласны с пользовательским соглашением

http://www. mathnet.ru/rus/agreement

Параметры загрузки:

IP : 34.239 .49 .27

26 апреля 2023 г., 13:51:12

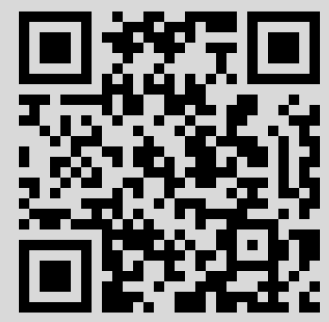




\section{СЕМЕЙСТВА ПРИВОДИМЫХ АБЕЛЕВЫХ ПОВЕРХНОСТЕЙ}

\section{И.Р. Шафаревич}

В работе доказывается, что для непостоянных семейств абелевых многообразий, определенных над кривыми, являющимися накрытиями заданной степени проективной прямой, общий член которых имеет число Пикара 3 , решетка Севери общего члена может принадлежать лишь к конечному числу типов, с точностью до изоморфизма.

Мы продолжаем здесь рассматривать вопрос, разбиравшийся в работах [1] и [2]. В работе [1] рассматривались абелевы поверхности, определенные над полями алгебраических чисел $K$, имеющими фиксированную степень $n$. В предположении, что число Пикара абелевой поверхности имеет максималњное значение 4 доказывалось, что решетка Севери поверхности может принадлежать лишь к конечному числу типов, с точностью до изоморфизма. Здесь мы рассмотрим функциональный аналог этого вопроса для следующего случая, когда число Пикара равно 3.

Пусть $\mathfrak{A} \rightarrow S$ - непостоянное семейство абелевых поверхностей над кривой $S$, общий член которого $A$ имеет число Пикара 3 . Пусть $S$ допускает непостоянньй морфизм $S \rightarrow \mathbb{P}^{1}$ заданной степени $n$.

Теорема. Число неизоморфных решеток Севери $S$ ограничено константой, зависящей только от $n$.

При условиях теоремы $A$ является или ложной эллиптической кривой, или приводимо и изогенно квадрату эллиптической кривой $E$. Первый случай рассмотрен в работе [2]. Здесь мы рассмотрим второй случай. Таким образом, $A$ изогенно $E \times E$. Мы будем записывать $E \times E$ в виде $E \times E^{\prime}$, где $x \rightarrow x^{\prime}$ - некоторый фиксированный изоморфизм двух экземпляров $E$. Нам достаточно доказать

ПРЕДЛОЖЕНИЕ. Число неизоморфных абелевых поверхностей, изогенных $E \times E^{\prime}$ и определенных над $\mathbb{C}(S)$, ограничено константой, зависящей только om $n$.

Нам понадобится аналог этого утверждения для одной кривой $E$.

ЛЕмма. Число неизоморфных эллиптических кривых, изогенных $E$ и определенных над $\mathbb{C}(S)$, ограничено константой, зависящей от $n$.

ДокАЗАТЕльство. Пусть $X_{0}(N)$ - модулярная кривая, параметризующая эллиптические кривые вместе с изогенией с циклическим ядром порядка $N$. Рассуждения, приведенные в работе [2], показывают, что достаточно ограничить значения $N$, для которых кривая $X_{0}(N)$ допускает морфизм степени $n$ на $\mathbb{P}^{1}$. Рассуждение в точности следует Оггу [3], который при $n=2$ даже перечислил все такие $N$. Кривая $X(N)$ определена над $\mathbb{Q}$. Как показано в работе [2], эта кривая допускает морфизм $f: X(N) \rightarrow C$ степени $\leqslant \nu$ на кривую $C$, определенную над $\mathbb{Q}$ и имеющую род $\leqslant(n / \nu-1), \nu \mid n$. Достаточно рассмотреть случай $N=p^{d}$ и ограничить $p$ и $d$. Кривая $X_{0}\left(p^{d}\right)$ имеет хорошую редукцию по любому простому модулю $l \neq p$, например, $l=2$ или 3 . Пусть, например, $l=2$. Над полем $\mathbb{F}_{4}$ существует суперсингулярная кривая. Для нее эндоморфизм $\Phi$ робениуса имеет вид $\varphi=-2$ Id. Поэтому

(С) И.Р. ШАФАРЕВИч 1996 
все изогении определены над $\mathbb{F}_{4}$. Таким образом, мы получаем $\frac{1}{24} p^{d-1}(p+1)$ точек на кривой $X_{0}\left(p^{d}\right) / 2$. С другой стороны, легко видеть, что эта кривая допускает морфизм $f$ на кривую $C$ с такими же свойствами, что и морфизм $f$. Применяя оценку Вейля к кривой $C$, мы получаем

$$
\#\left(\frac{X_{0}\left(p^{d}\right)}{2}\right)\left(\mathbb{F}_{4}\right) \leqslant \nu\left(5+4\left(\frac{n}{\nu}-1\right)^{2}\right)
$$

т.e.

$$
\frac{1}{24} p^{d-1}(p+1) \leqslant \nu\left(5+4\left(\frac{n}{\nu}-1\right)^{2}\right),
$$

что и доказывает лемму.

ДОКАЗАТЕЛЬСТВО ПРЕДЛОЖЕНИЯ. Начнем с ряда редукций. МЫ долЖны оценить число неизоморфных среди поверхностей $\left(E \times E^{\prime}\right) / M$, где \# $M=p^{d}$ и $M$ определено над полем $\mathbb{C}(S)$. Если $M \supset E_{p}$, то $\left(E \times E^{\prime}\right) / M \simeq\left(E \times E^{\prime}\right) / M^{\prime}$, где $M^{\prime}=M / E_{p}$, и задача сводится к меньшему $M$. Аналогично при $M \supset E_{p}^{\prime}$. Поэтому мы можем считать, что $M \cap E=\{\alpha\}, M \cap E^{\prime}=\left\{\beta^{\prime}\right\}, p^{r} \alpha=0, p^{s} \beta^{\prime}=0$, причем согласно лемме $p^{r} \leqslant c, p^{s} \leqslant c$, где $c$ - константа, зависящая только от $p^{d}$. Как всякая подгруппа прямого произведения, $M$ может быть получено как прообраз графокиа $\Gamma \varphi$, где $\varphi$ - изоморфизм подгрупп $A \subset E /\{\alpha\}$ и $A^{\prime} \subset E^{\prime} /\left\{\beta^{\prime}\right\}$. Очевидно, что существует изогения $\chi: E^{\prime} /\left\{\beta^{\prime}\right\} \rightarrow E /\{\alpha\}$, степень которой не превосходит $c^{2}$. Положим $\chi \varphi=\varphi^{\prime}, \varphi^{\prime}: A \rightarrow E /\{\alpha\}$. Мы получаем изогению

$$
\left(E /\{\alpha\} \times E /\left\{\beta^{\prime}\right\}\right) / \Gamma_{\varphi} \rightarrow(E /\{\alpha\} \times E /\{\alpha\}) / \Gamma_{\varphi^{\prime}}
$$

степень которой также ограничена в зависимости от $p^{d}$. Решетки Севери двух таких абелевых поверхностей определяют друг друга с точностью до конечного числа возможностей, ограниченных в зависимости от $p^{d}$. Заменяя $E /\{\alpha\}$ на $E$, мы редуцируем общий случай к случаю $M=\Gamma_{\varphi}$, где $\varphi: A \rightarrow E^{\prime}$ - гомоморфизм подгруппы $A \subset E$, определенной над полем $\mathbb{C}(S)$. Пусть теперь подгруппа $A$ имеет тип $\left(p^{u}, p^{v}\right), u \leqslant v$. Тогда $p^{v-u} A-$ циклическая подгруппа, определенная над $\mathbb{C}(S)$, и согласно лемме $p^{v-u} \leqslant c$. Обозначим через $\bar{\varphi}$ ограничение $\varphi$ на $E_{p^{u}}$. Тогда $\left(\Gamma_{\varphi}: \Gamma_{\bar{\varphi}}\right) \leqslant c$, и мы имеем изогению $\left(E \times E^{\prime}\right) / \Gamma_{\bar{\varphi}} \rightarrow\left(E \times E^{\prime}\right) / \Gamma_{\varphi}$ степени $\leqslant c$. Этим утверждение сводится к случаю $A=E_{p^{u}}, \varphi \subset$ End $E_{p^{u}}$.

Наконец, предположим, что $\varphi$ представляется в виде $\varphi=a \operatorname{Id}+p \psi, a \in \mathbb{Z}$. Положим $E(a)=\{(x, a x), x \in A\}$. Очевидно, что $E(a) \simeq E$ и $E \times E^{\prime}=E(a) \times E^{\prime}$. В последнем представлении эндоморфизм $\varphi$ записывается в виде $(0, p \psi)$. Так как $\Gamma_{p \psi} / E_{p}=\Gamma_{\bar{\psi}}$, где $\bar{\psi}$ рассматривается как эндоморфизм группы $E_{p^{u}} / E_{p} \simeq E_{p^{u-1}}$ и

$$
\left(E \times E^{\prime}\right) / \Gamma_{p \psi}=\left(\left(E \times E^{\prime}\right) / E_{p}\right) /\left(\Gamma_{p \psi} / E_{p}\right) \simeq\left(E \times E^{\prime}\right) / \Gamma_{\bar{\psi}},
$$

то рассмотрение эндоморфизма $\varphi$ группы $E_{p}$ сводится к рассмотрению эндоморфизма $\bar{\psi}$ группы $E_{p^{u-1}}$. Таким образом, мы можем предполагать, что

$$
\varphi \neq a \mathrm{Id}+p \psi
$$


Теперь можно опять применить прием Огга из его работы [3], использованньй уже при доказательстве леммы и в работе [2]. Мы рассматриваем структуры, состоящие из эллиптической кривой $E$ и эндоморфизма $\varphi$ группы $E_{p} u$. Их параметризация описана в работе Делиня и Раппопорта [4]. При $p>3$ они описьваются кривой, имеющей гладкую модель над $\mathbb{Z}[1 / p]$. Эта кривая распадается на неприводимые компоненты, соответствующие классам сопряженности эндоморфизмов относительно $\operatorname{Aut}\left(E_{p^{u}}\right)$. Каждая неприводимая компонента является "модулярной кривой уровня $H$ " - $M_{H}$ - в смысле работы [4], где $H$ - централизатор соответствующего эндоморфизма в группе $\mathrm{GL}\left(2, \mathbb{Z} / p^{u}\right)$. Согласно [4] кривая определена над подполем кругового поля $\mathbb{Q}\left(\zeta_{p^{u}}\right)$, принадлежащем группе автоморфизмов, возводящих $\zeta_{p} u$ в степени $\operatorname{det} g, g \in H$. Так как $H$ в нашем случае содержит все гомотетии, то эта группа содержит $\left(\left(\mathbb{Z} / p^{u}\right)^{x}\right)^{2}$, а соответствующее поле квадратично или композит двух квадратичных. Поэтому поле классов вычетов по простому идеалу, делящему простое число $l$, содержит не более чем $l$ элементов.

Теперь применим прием Огга и рассуждения из работы [2]. С одной стороны, так как семейство $\left(E \times E^{\prime}\right) / \Gamma_{\varphi}$ определено над кривой $S$, имеющей морфизм $S \rightarrow \mathbb{P}^{1}$ степени $p^{u}$, мы получаем, что таким же морфизмом обладает кривая $M_{H}$. Отсюда вытекает существование морфизма $M_{H} \rightarrow C$ с теми же свойствами, что и в доказательстве леммы (как уже было доказано в работе [2]). Выбирая простое число $l=2$ или 3 , отличное от $p$, мы получаем, как при доказательстве леммы (или в работе [2]), неравенство для числа точек кривой $M_{H} / \mathfrak{l}$ (где $\mathfrak{l}-$ простой делитель $l$ в поле определения кривой $M_{H}$ ). В поле $\mathbb{F}_{4}$ (полагая для определенности $l=2$ )

$$
\#\left(M_{H} / \mathfrak{l}\right)\left(\mathbb{F}_{4}\right) \leqslant \nu\left(5+4\left(\frac{n}{\nu}-1\right)^{2}\right) .
$$

С другой стороны, то, что структура $(E, \varphi)$ определена над полем $K$, означает, что кривая $E$ определена над этим полем, и

$$
\varphi \sigma=\sigma \varphi
$$

для автоморфизмов $\sigma$ группы Галуа алгебраическогозамыкания этого поля. В частности, если кривая $E$ определена над полем $\mathbb{F}_{4}$ и суперсингулярна, то автоморфизмы группы Галуа алгебраического замыкания действуют как гомотетии и поэтому все структуры $(E, \varphi)$ определены над полем $\mathbb{F}_{4}$ и дают точки из $\left(M_{H} / \mathfrak{l}\right)\left(\mathbb{F}_{4}\right)$. Число разных структур равно $\left(\mathrm{GL}\left(2, \mathbb{Z} / p^{u}\right): H\right)$. Поэтому

$$
\#\left(M_{H} / \mathfrak{l}\right)\left(\mathbb{F}_{4}\right) \geqslant\left(\mathrm{GL}\left(2, \mathbb{Z} / p^{u}\right): H\right)
$$

и нам остается дать оценку снизу для последнего индекса.

Мы будем считать, что группа $\operatorname{GL}\left(2, \mathbb{Z} / p^{u}\right)$ действует на группе $\left(\mathbb{Z} / p^{u}\right)^{2}$. Сначала докажем, что для рассматриваемого эндоморфизма $\varphi$ можно найти такой элемент $e \in\left(\mathbb{Z} / p^{u}\right)^{2}$, что $e$ и $\varphi(e)$ порождают всю группу $\left(\mathbb{Z} / p^{u}\right)^{2}$. Если $\varphi$ задается матрицей $\left(\begin{array}{ll}a & b \\ c & d\end{array}\right)$, то для этого достаточно найти такие $u$ и $v \in \mathbb{Z} / p^{u}$, что

$$
\operatorname{det}\left(\begin{array}{cc}
u & v \\
a u+c v & b u+d v
\end{array}\right) \not \equiv 0 \quad \bmod p
$$

т.е. $b u^{2}+(d-a) u v-c v^{2} \not \equiv 0 \bmod p$. Они существуют, если хоть один из вычетов $b$, $(d-a), c \bmod p$ отличен от 0 . В нашем случае это так, поскольку мы предполагаем, что эндоморфизм $\varphi$ не представим в виде $a \operatorname{Id}+p \psi($ cp. (1)). 
Таким образом, $\varphi$ реализуется как умножение на $T$ в модуле $\left(\mathbb{Z} / p^{u}\right)[T] / F$, где $F(T)$ - характеристический многочлен эндоморфизма $\varphi$. Легко видеть, что в этом случае централизатор $\varphi$ (т.е. $H)$ совпадает с $\left(\left(\mathbb{Z} / p^{u}\right)[T] / F\right)^{x}$. Порядок последней группы вычисляется очевидньм образом: надо рассмотреть гомоморфизм

$$
\left(\mathbb{Z} / p^{u}\right)[T] / F \rightarrow(\mathbb{Z} / p)[T] / \bar{F} .
$$

Очевидно, что элемент слева обратим тогда же, когда и его образ справа. Поэтому

$$
\#\left(\left(\mathbb{Z} / p^{u}\right)[T] / F\right)^{x}=p^{2 u-2} \#((\mathbb{Z} / p)[T] / \bar{F}) .
$$

В случаях, когда многочлен $\bar{F}$

a) разлагается на два разных линейных множителя над $\mathbb{F}_{p}$,

b) является квадратом линейного и

c) неприводим над $\mathbb{F}_{p}$,

$\#((\mathbb{Z} / p)[T] / \bar{F})$ равняется a) $\left.\left.(p-1)^{2}, \mathrm{~b}\right)(p-1) p, \mathrm{c}\right) p^{2}-1$, и, значит, \# $\left(\left(\mathbb{Z} / p^{u}\right)[T] / F\right)$ равняется а) $\left.\left.(p-1)^{2} p^{2 u-2}, \mathrm{~b}\right)(p-1) p^{2 u-1}, \mathrm{c}\right)\left(p^{2}-1\right) p^{2 u-2}$. Так как

$$
\# \mathrm{GL}\left(2, \mathbb{Z} / p^{u}\right)=\left(p^{2}-1\right)(p-1) p^{4 u-3},
$$

то $\left(\mathrm{GL}\left(2, \mathbb{Z} / p^{u}\right): H\right)$ равняется a) $p^{2 u-1}(p+1)$, b) $\left.p^{2 u-2}\left(p^{2}-1\right), \mathrm{c}\right) p^{2 u-1}(p-1)$. Во всех случаях это число $\geqslant p^{2 u-1}$. Вместе с неравенством (2) это дает ограничение на $p^{u}$, т.е. доказывает предложение, $\mathrm{a}$, значит, и теорему.

ЗАмЕчАнИЕ 1. Из доказанной теоремы следует аналогичное утверждение о семействах поверхностей типа КЗ с числом Пикара 19. Редукция к случаю абелевых многообразий дословно такая же, как в случае числа Пикара 20, проведенная в работе [1].

ЗАмеч Ание 2. А. Н. Паршин обратил мое внимание на то, что лемма, доказанная в начале работы, была также доказана в препринте [5].

ЗАмечАниЕ 3. Результат, аналогичный доказанному в лемме, для случая числовых полей недавно получил Мерел. Вопрос в этом случае гораздо труднее, но и оценка порядка возможной цик лической подгруппы $A \subset E(K)$ порядка $p^{u}$, определенной над полем $K$, в зависимости от степени поля $K$, слабее: она экспоненциальна, в то время, как из доказательства леммы вытекает квадратичная оценка. Было бы интересно найти истинную оценку в функциональном случае. Может ли она быть линейной?

Математический институт

Поступило им. В.А. Стеклова РАН 14.08 .96

\section{СПИСОК ЦИТИРОВАННОЙ ЛИТЕРАТУРЫ}

1. Shafarevich I. R. // Algebra and Analysis / ed. M. M. Arslanov: De Gruyter, 1996. Р. 103-108. 2. Шафаревич И. Р. // Изв. РАН. Сер. матем. 1966. Т. 60. №5. C. 213-223. 3. Ogg A. // Bull. Soc. Math. France. 1974. V. 102. P. 449-462. 4. Deligne P., Rappoport M. // Lecture Notes in Math. 1972. V. 349. P. 143-316. 5. Khae Viet Nguen, Masa-Hiko Saito. $D$-gonality of Modular Curves and Bounding Torsion. Preprint: Kyoto Univ., 1996. March. 